\title{
Original Research \\ Canonical WNT pathway inhibition reduces ATP synthesis rates in glioblastoma stem cells
}

\author{
Dymphna Margriet Ouwens ${ }^{1,2,3}$, Michael Hewera ${ }^{4}$, Guanzhang $\mathrm{Li}^{5}$, Wang $\mathrm{Di}^{5}$, \\ Sajjad Muhammad $^{4}$, Daniel Hänggi ${ }^{4}$, Hans-Jakob Steiger ${ }^{6}$, Claudia A. Dumitru ${ }^{7}$, \\ Erol Sandalcioglu ${ }^{7}$, Roland S Croner ${ }^{8}$, Wei Zhang ${ }^{5}$, Or Kakhlon ${ }^{9}, *, \dagger$, Ulf D. Kahlert ${ }^{8, *, \dagger}$ \\ ${ }^{1}$ Institute for Clinical Biochemistry and Pathobiochemistry, German Diabetes Center (DDZ), 40225 Düsseldorf, Germany \\ ${ }^{2}$ German Center for Diabetes Research (DZD), München-Neuherberg, 40225 Düsseldorf, Germany \\ ${ }^{3}$ Department of Endocrinology, Ghent University Hospital, 41045 Ghent, Belgium \\ ${ }^{4}$ Clinic of Neurosurgery, University Hospital Düsseldorf and Medical Faculty, Heinrich Heine University, 40225 Düsseldorf, Germany \\ ${ }^{5}$ Beijing Neurosurgical Institute, Capital Medical University, 100054 Beijing, China \\ ${ }^{6}$ Department of Neurosurgery, Canton Hospital Aarau, 5001 Aarau, Switzerland \\ ${ }^{7}$ Clinic for Neurosurgery, Medical Faculty and University Hospital Magdeburg, 39120 Magdeburg, Germany \\ ${ }^{8}$ Molecular and Experimental Surgery, Clinic for General, Visceral, Vascular, and Transplant Surgery, Medical Faculty and University Hospital \\ Magdeburg, 39120 Magdeburg, Germany \\ ${ }^{9}$ Department of Neurology, Hadassah-Hebrew University Medical Center, Ein Kerem, 91120 Jerusalem, Israel \\ *Correspondence: Ulf.kahlert@med.ovgu.de (Ulf D. Kahlert); ork@hadassah.org.il (Or Kakhlon) \\ $\dagger$ These authors contributed equally. \\ Academic Editor: Graham Pawelec \\ Submitted: 30 September 2021 Revised: 13 December 2021 Accepted: 27 December 2021 Published: 18 January 2022
}

\begin{abstract}
Background: The conserved stem cell signaling network canonical Wingless (WNT) plays important roles in development and disease. Aberrant activation of this pathway has been linked to tumor progression and resistance to therapy. Industry and academia have substantially invested in developing substances, which can efficiently and specifically block the WNT signaling pathway. However, a clear clinical proof of the efficacy of this approach is still missing. Studies on the metabolomics dysregulation of cancer cells have led to innovations in oncological diagnostics. In addition, modulation of cancer cell metabolome is at the base of promising clinical oncology trials currently underway. While onco-protein activation can have profound metabolic outcomes, the involvement of stem cell signals, such as the WNT pathway, in tumor cell metabolomics is yet insufficiently characterized. Material and methods: We determined live cell metabolism and bioenergetics in pathophysiological relevant, WNT-dependent glioblastoma stem cell (GSC) models. We quantified those parameters in cells with canonical WNT activity and in isogenic cells where WNT activity had been inhibited by short hairpin RNA against $\beta$-catenin. Furthermore, we applied computational analysis of RNA sequencing to verify our functional findings in independent GSCs cohorts. Results: The investigated collection of disease models allows the separation in tumors with low, moderate and high base line metabolic activity. Suppression of canonical WNT signaling led to significant reduction of total, mitochondrial, and glycolytic ATP production rates. Elevated canonical WNT transcription signature in GSCs positively correlated with transcription levels of mitochondrial ATP synthesis, whereas non-canonical WNT gene expression signature did not. Conclusion: The applied disease modeling technology allows the recapitulation of inter-tumoral heterogeneous metabolic properties of glioblastoma. Our data show for the first time that inhibition of canonical WNT signaling in alive GSCs functionally correlates with energy inhibition and glucose homeostasis. As this correlation occurs in GSCs from different transcriptional or epigenetic transcriptional subtypes, our results suggest that developing therapies directed against glycolysis/ATP-synthesis may be a promising strategy to overcome therapy resistance due to inter-tumoral heterogeneity and offers starting point to impair downstream signal WNT.
\end{abstract}

Keywords: Tumor stem cells; WNT; Metabolism; Bioenergetics; Single cell RNA sequencing

\section{Introduction}

Accumulating evidence demonstrates that the Wingless (WNT) network - one of the phylogenetically most conserved developmental signaling pathways - acts as an oncogenic driver in cancers $[1,2]$. Various WNT inhibitors are currently being tested in clinical trials [3]. However, not a single WNT inhibitor has been approved for cancer therapy thus far. Previous clinical studies showed that systemic administration of current WNT blockers resulted in intolerable side effects, usually due to targeting the WNT niche in the gastrointestinal tract [1]. The identification of a putative blocker of cancer-specific WNT signaling would be a promising strategy to overcome this hurdle.

The metabolic profile of cancer cells significantly differs from that of non-neoplastic cells. Some cancer cells are abnormally dependent on energy production through anaerobic oxidation and show atypical activation of glutaminolysis as well as fatty acid production [4]. It was suggested that 
therapeutic resistance is the consequence of these metabolic alterations, which represent a major obstacle in the treatment of cancer [5]. Several studies reported that high activity of glycolysis causes chemotherapy resistance in aggressive cancer cells [6]. Moreover, there is increasing evidence that aggressive cancer cells in various tissues including brain, can re-program their metabolism in response to altered intrinsic and environmental conditions [7-10].

The impact of WNT signaling on the cellular energy status of tumor cells has been investigated to some extent. However, limited knowledge is available regarding its involvement in brain tumor bioenergetics. WNT signaling activates the potent proto-oncogene MYC in a variety of cancers [11], which subsequently promotes anaerobic respiration and glutaminolysis [12]. WNT was found to promote glycolysis in breast [13] and colon [14] cancer. A prominent oncogenic driver, also present in gliomas, is epidermal growth factor receptor (EGFR) signaling, which induces translocation of the glycolysis mediator pyruvate kinase isoform M2 (PKM2) to the cell nucleus, where it directly binds to the key WNT transcription factor $\beta$-catenin. The effects on glycolytic activity in this model are currently unknown [15]. In breast cancer, metabolic stress (reactive oxygen species production) has been demonstrated to modulate WNT activity through interaction with $\beta$-catenin [16]. Furthermore, WNT signaling promotes breast cancer stemness by increasing mitochondrial mass and oxidative phosphorylation (OXPHOS) [17]. Another metabolic control of WNT signaling is related to nutrient levels. Specifically, over-nutrition stress - induced either genetically or by ad libitum feeding - stimulates WNT thereby enhancing adipogenesis [18]. Concordantly, non-esterified fatty acids are essential for post-translational maturation of the WNT ligands (palmitoylation) [19], which is essential for GSC survival [20]. In glioma, previous studies showed that EHZ2the core component of PRC2- functions as a histone methyltransferase for the trimethylation of histone 3 on lysine 27 (H3K27me3). EHZ2 disruption can suppress aerobic glycolysis in glioblastoma cells, accompanied by inhibition of $\beta$ catenin transcription [21]. Moreover, dysregulation of thermodynamics and circadian rhythm in glioma cells was identified by metabolic changes in response to WNT activation [22]. Recently, purine metabolism was found to be regulated in glioblastoma stem cells (GSCs) under acidic stress conditions characteristic of the core region of rapidly growing tumor parenchyma. However, no association to WNT signaling was recorded [23].

The present study used glioblastoma as a cancer stem cell model since this tumor type is known to harbor a WNTdriven subpopulation of stem cells [24,25]. Capitalizing on genetic engineering, gene expression and live cell bioenergetic analyses, we aimed at identifying the effect of WNT signaling on GSC metabolism. We found that GSC cell lines manifested variable overall ATP production rates to which mitochondrial oxidative phosphorylation and gly- colysis contributed equally. Interestingly, suppression of WNT signaling by $\beta$-catenin (CTNNB1) knockdown reduced total, mitochondrial and glycolytic ATP production rates in most GSC lines. These data are in agreement with single cell mRNAseq analysis showing that canonical and overall WNT signaling gene expression positively correlates with the expression of genes involved in the activation of the mitochondrial electron transport chain. Thus, our study contributes towards elucidation of cancer stem cell metabolism. Since WNT signaling, which is key to GSC therapy resistance, is also essential for the coordinated bioenergetics and metabolism of GSCs of different molecular subtypes [26], anti-ATP-synthesis directed therapies might be particularly successful in overcoming therapy resistance associated with tumor heterogeneity.

\section{Material and methods}

\subsection{Patients and their derived GSC samples for sequencing analysis}

The collection of glioma samples was approved by the Beijing Tiantan Hospital institutional review board (IRB) and written informed consent forms were obtained from every patient. The site of tumor sample collection was planned in the neuronavigation software before surgery. Following their surgical removal, tumor tissues were immediately immersed in culture medium and transported to the laboratory within $30 \mathrm{~min}$. Glioma cells, obtained from these primary tumors according to Zhai et al. [27], were cultured in DMEM/F12 serum-free medium (Gibco) supplemented with B27 (Gibco), basic fibroblast growth factor (BFGF, $20 \mathrm{ng} / \mathrm{mL}$ ), epidermal growth factor (EGF, $20 \mathrm{ng} / \mathrm{mL}$ ), and heparin $(2.5 \mathrm{mg} / \mathrm{mL})$. Cells were regularly passaged according to their growth status. GSC gene expression array data was downloaded from GSE67089 (https://www.ncbi.nlm.n ih.gov/geo/query/acc.cgi?acc=GSE67089).

\subsection{Transcriptome sequencing}

Total RNA was extracted using the mRNA Isolation Kit (Ambion, TX, USA) following manufacturer instructions. RNA integrity was evaluated by Bioanalyzer and samples with RNA Integrity Number $\geq 7$ were selected for further transcriptome sequencing. The libraries were constructed using TruSeq Stranded mRNA LTSample Prep Kit and sequenced on the Illumina sequencing platform (HiSeqTM 2500 or Illumina HiSeq X Ten).

\subsection{Gene set variation analysis (GSVA)}

The gene list of biological processes was obtained from the GSEA Web portal (http://software.broadinstitut e.org/gsea/msigdb/index.jsp). Based on the transcriptome sequencing data, the biological enrichment score of each tumor sample was obtained through the GSVA algorithm. The GSVA algorithm was performed by $\mathrm{R}$ under the default parameters. 


\subsection{Correlation analysis}

The correlation between biological function enrichment scores were evaluated by the Pearson correlation analysis. Correlation plots were drawn by the ggplot 2 package (RStudio Inc, MA, USA) in R language.

\subsection{Cell culture}

GBM1, 233, 644 and JHH520 cells were cultured in DMEM/F12 (Gibco), supplemented with B27 (Gibco), basic fibroblast growth factor (Peprotech BFGF, $20 \mathrm{ng} / \mathrm{mL}$ ), epidermal growth factor (Peprotech EGF, $20 \mathrm{ng} / \mathrm{mL}$ ), and heparin sodium salt (Sigma $2.5 \mathrm{mg} / \mathrm{mL}$ ). Cells were tested for mycoplasma before each experiment. Incubated at 37 ${ }^{\circ} \mathrm{C}$ and $5 \% \mathrm{CO}_{2}$, the cells were passaged whenever they reached $80 \%$ density. The following models were kindly provided by our colleagues: 233 (= BTSC233) and 407 (= BTSC407) from M.S. Carro, Freiburg University, Freiburg im Breisgau, Germany; JHH520 from G. Riggins, Johns Hopkins, Baltimore, MD, USA; 644 (= NCH644) from C. Herold-Mende, Heidelberg University, Heidelberg, Germany, GBM1 (= HSR-GBM1) from A. Vescovi, Milan, Italy; and SF188 from E. Raabe, Johns Hopkins University, Baltimore, MA, USA). Ethical approval to conduct the in vitro studies was granted by the ethics committee of the Medical Faculty of the Heinrich-Heine University (study ID 5841R).

\subsection{Genetic WNT inhibition model}

HEK $293 \mathrm{~T}$ cells were cultured in $10 \mathrm{~cm}$ dishes with DMEM (Gibco) supplemented with 10\% FBS (SigmaAldrich) and 1\% Penicillin/Streptomycin (Sigma) until they reached $80 \%$ confluence. Subsequently, the medium was replaced with antibiotic free DMEM/FBS. Cells where transfected with transfection mix (800 $\mu \mathrm{L}$ DMEM, $4 \mu \mathrm{L}$ pMDL g/pRRE (Addgene \#12259), $2 \mu \mathrm{L}$ pRSV-Rev (Addgene \#12253), $2 \mu \mathrm{L}$ pMD2.G (Addgene \#12259), $8 \mu \mathrm{L}$ pLKO.1 puro EV (\#8453) or pLKO.1 puro shBeta-Catenin and $60 \mu \mathrm{L}$ FuGene transfection reagent (Promega \#E2311). On day 1 after transfection, the medium was replaced by DMEM/FCS/P-S again. During days 2-4 after transfection, $10 \mathrm{~mL}$ supernatant was collected through $0.45 \mu \mathrm{L}$ filter into $15 \mathrm{~mL}$ tubes containing $1 \mathrm{~mL} \mathrm{50 \%} \mathrm{PEG} \mathrm{(Sigma-Aldrich,}$ MO, USA). Then $900 \mu \mathrm{L}$ of $1.5 \mathrm{M} \mathrm{NaCl}$ was added. Tubes were then vortexed and stored overnight at $4{ }^{\circ} \mathrm{C}$. On the next day, tubes were centrifuged at $7000 \mathrm{~g}$ and $4{ }^{\circ} \mathrm{C}$ for 30 min. Next, the supernatant was removed without disturbing the virus particle pellets. Pellets were then resuspended in $150 \mu \mathrm{L}$ PBS. The suspension was then aliquoted into 1.5 $\mathrm{mL}$ tubes $(50 \mu \mathrm{L}$ each).

For transduction, approx. $5 \times 10^{5}$ wild type cells where centrifuged in $15 \mathrm{~mL}$ tubes, supernatant was removed and $15 \mu \mathrm{L}$ of virus particles were added to the pellet. Then the pellet was resuspended and transferred into one well of a 6 well plate (Greiner). Each wildtype cell line (WT) was transfected with pLKO shRNA against $\beta$ -
Catenin (shCTNNB1) and pLKO empty vector (EV). On day 1 after transduction, $1 \mathrm{~mL}$ of medium was added to each well. On day 3 after transduction, $3 \mathrm{~mL}$ medium where added and puromycin (Gibco) was added to each well to select the cells $(20 \mu \mathrm{g} / \mathrm{mL})$. On day 5 after transduction, medium was replaced with fresh medium containing puromycin. On day 7 that procedure was repeated. On day 9 after transduction, the medium was changed to normal medium and the cells were then ready to be used in downstream experiments.

To validate the model by target activation, we performed Western blot analysis. Cell suspensions were centrifuged, and pellets where lysed with cell lysis buffer (50 $\mathrm{mM}$ TRIS-HCl (pH 8), $150 \mathrm{mM} \mathrm{NaCl}, 0.5 \%$ Triton X$100,0.5 \%$ Deoxycholate) with a general use protease inhibitor cocktail for $30 \mathrm{~min}$ on ice. The lysates were centrifuged at $13000 \mathrm{rpm}$ at $4{ }^{\circ} \mathrm{C}$ for $10 \mathrm{~min}$ to pellet DNA. The supernatants were transferred into new $1.5 \mathrm{~mL}$ tubes. Protein concentration was measured using the BCA Assay (Bio-Rad) with BSA Standards (Bio-Rad). $30 \mu \mathrm{g}$ of protein were loaded onto $4-15 \%$ gradient precast BAA gels (Bio-Rad). SDS-PAGE was performed at $110 \mathrm{~V}$ for $60 \mathrm{~min}$. Transfer onto nitrocellulose membranes (General Electric Healthcare, IL, USA) was performed at 250 $\mathrm{mA}$ for $2 \mathrm{~h}$. Subsequently, the membranes were blocked with 5\% BSA in TBS-T for $1 \mathrm{~h}$ and then incubated with 1:500 purified mouse Anti- $\beta$-Catenin antibodies (BD Biosciences, NJ, USA, \#610153), 1:1000 rabbit anti- $\beta$ Actin antibodies (Cell Signaling Technology, MA, USA, \#4970) and 1:10000 GAPDH monoclonal antibodies (Proteintech, Tokyo, \#60004-1-1 g) in 5\% BSA in TBS-T at $4{ }^{\circ} \mathrm{C}$, overnight. For visualization, the membranes were incubated with IRDye 680RD Donkey anti-Mouse IgG antibodies (1:10000, LiCor \#926-68072) or IRDye800CW goat-anti-rabbit IgG antibodies (1:10000, LI-COR, \#92632211 ) for $1 \mathrm{~h}$ at room temperature and then scanned with an Odyssey DLX Imaging System (LiCor). PageRuler Plus Prestained Protein Ladder (Thermo Fisher, MA, USA, \#26620) was used as molecular weight marker. Protein levels were quantified by Image J (NIH, MD, USA).

\subsection{Extracellular flux analysis}

GBM1, 233 and JHH520 wildtype (wt), EV and sh $\beta$-Catenin (CTNNB1) cells were centrifuged and seeded in XF96e microplates (Agilent Technologies, Inc., Santa Clara, CA, USA) at a density of 25.000 cells per well. Per cell line, 6 wells were used and empty wells were filled with tissue culture medium. Following overnight incubation at $37{ }^{\circ} \mathrm{C}$ and $5 \% \mathrm{CO}_{2}$, the cultures were washed twice with Seahorse XF DMEM medium containing $5 \mathrm{mM}$ HEPES (Agilent Technologies) supplemented with $10 \mathrm{mM}$ glucose, $2 \mathrm{mM}$ glutamine and $1 \mathrm{mM}$ sodium pyruvate. Following a $1 \mathrm{~h}$ incubation in a $\mathrm{CO}_{2}$-free incubator at $37^{\circ} \mathrm{C}$, cells were placed in the XF96e extracellular flux analyzer (Agilent Technologies) for real time cell-based bioenergetic analy- 
sis, according to Aglilent's Seahorse Real-Time ATP Rate Assay kit and protocol. In brief, at baseline, the cellular oxygen consumption rates (OCR) and extracellular acidification rates (ECAR) were recorded four times at 6 min intervals. This was followed by the subsequent injections of $1.5 \mu \mathrm{mol} / \mathrm{L}$ oligomycin A, $0.5 \mu \mathrm{mol} / \mathrm{L}$ rotenone/antimycin $\mathrm{A}$, and, where indicated, $50 \mathrm{mM}$ 2-deoxyglucose. After each injection, the changes in OCR and ECAR were recorded three times at 6 min intervals. Oligomycin A, rotenone and antimycin A were purchased from Sigma Aldrich (St. Louis, USA) and dissolved in DMSO. Stocks of glucose, pyruvate and 2-deoxyglucose (all from Sigma Aldrich) were prepared in Seahorse XF DMEM medium. All stocks were aliquoted and stored at $-20{ }^{\circ} \mathrm{C}$ until use. The medium was aspirated and the cells were lysed in 50 $\mu \mathrm{L} 20 \mathrm{mM}$ Tris-HCl, $1 \mathrm{mM}$ EDTA, $1 \mathrm{mM}$ EGTA, $150 \mathrm{mM}$ $\mathrm{NaCl}, 1 \%$ Triton $\mathrm{X}-100, \mathrm{pH}$ 7.5. Following centrifugation for $5 \mathrm{~min}$ at $1000 \mathrm{rpm}$, protein content in the cell lysates was determined using the Pierce ${ }^{\mathrm{TM}} \mathrm{BCA}^{\mathrm{TM}}$ protein assay (Thermo Fisher Scientific, Waltham, MA, USA). The extracellular flux data were normalized for protein content and analyzed by the WAVE software (version 2.6.0.31, Agilent Technologies). Using this software, the ECAR values were converted to proton extrusion rates (PER). The protein and baseline-adjusted OCR and PER values were used to calculate ATP production rates: mitochondrial and glycolytic ATP production, as described elsewhere [28,29]. Formulas used to calculate the metabolic/bioenergetics parameters can be found in the supplementary data.

\subsection{Statistical analysis of functional assays}

The box and whisker plots show the median and 595\% confidence interval. Significant differences among cell lines were determined by one-way ANOVA followed by Sidak analysis for multiple comparisons. $p$-values of $<0.05$ were considered statistically significant. Statistical analysis was conducted by GraphPad Prism 9.2.0 (GraphPad Software, San Diego, CA, USA).

\section{Results}

\subsection{Metabolic profiling of GSC models}

We applied extracellular flux analysis to quantify ATP production rates in living cells. Fig. 1A shows that the JHH520 and 233 GSC lines displayed the highest ATP production rates followed by GBM1 and 644. The lowest rates of ATP production were observed in 407 and SF188 cells. The differences in total ATP production rates could be ascribed to changes in both mitochondrial and glycolytic ATP production (Fig. 1B,C). Yet, the two bioenergetic modes equally contributed to total ATP production, as demonstrated by the similarity of glycolytic to mitochondrial ATP ratios among the different cell lines (Fig. 1D).

\subsection{Impact of $\beta$-catenin knockdown on ATP production} rates

To explore the impact of canonical WNT signaling on mitochondrial and glycolytic function, we created stable cell lines modeling loss of function of WNT signaling [30]. The efficiency of $\beta$-catenin knockdown (CTNNB1 KD) in these cells was determined by Western blot (Fig. 2A). We used $\beta$-actin (ACTB) as loading control, since this cytoskeleton protein should not be affected by changes in cellular metabolism and bioenergetics and is considered a suitable normalization gene for many tissues [31]. CTNNB1 $\mathrm{KD}$ decreased total ATP production rates by up to $75 \%$ in all cell lines ( $p<0.001$, ANOVA) (Fig. 2B). This effect could be attributed to reductions of both mitochondrial and glycolytic ATP production rates.

Due to technical issues, we used GAPDH as a loading control for cell line 233. GAPDH is not an ideal loading control as it is a glycolytic enzyme, which could be influenced by WNT signaling. However, the fact that glycolytic ATP production was decreased by CTNNB1 KD in the 233 cells, while GAPDH did not change (Supplementary Fig. 1), suggest that GAPDH was not modified by CTNNB1 KD and thus could serve as a proper loading control.

\subsection{Correlation between WNT signaling and ATP synthesis pathways in GSCs}

We next tested whether the functional regulation of ATP synthesis by canonical WNT signaling is also mirrored by correlations at gene expression level. This was done using an in silico analysis of datasets derived from GSCs. We assessed single cell RNA sequencing of GSCs retrieved from different datasets and found a significant positive correlation between the expression of ATP synthesis genes and genes belonging to canonical WNT activation. In contrast, the expression of ATP synthesis genes did not correlate with the non-canonical WNT pathway (Fig. 3).

\section{Discussion}

Although cancer metabolism is a global and very active research field, metabolic characterization of physiologically relevant molecular subtypes of tumor stem cells, including GSCs, is insufficiently described [32]. Our approach, based on investigating major embryonic stem cell pathways and living cell populations, can potentially bridge this knowledge gap.

WNT-dependent GSCs manifest variable baseline ATP synthesis rates, reflecting the ability of our disease modeling technology to recapitulate wide spectra of the cellular and genetic tumor heterogeneity. Our results demonstrate that inhibition of canonical WNT signaling by CTNNB1 knock-down can suppress the mitochondrial and glycolytic activity in these cells. Notably, our previous studies showed that the potential of pharmacological WNT inhibition (using the Novartis drug candidate WNT974) is independent of the level of baseline WNT 
A

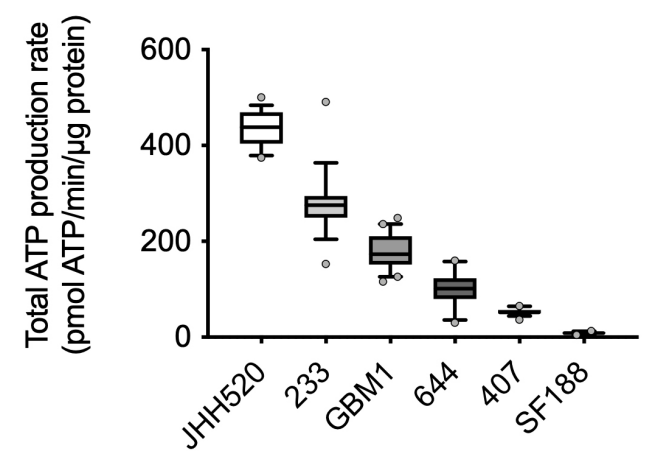

C

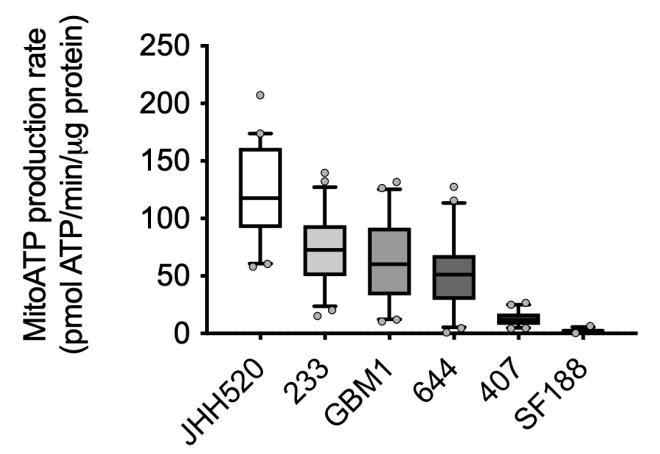

B

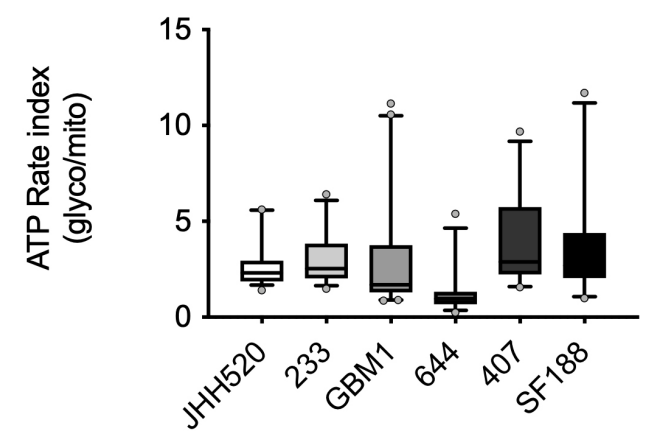

D

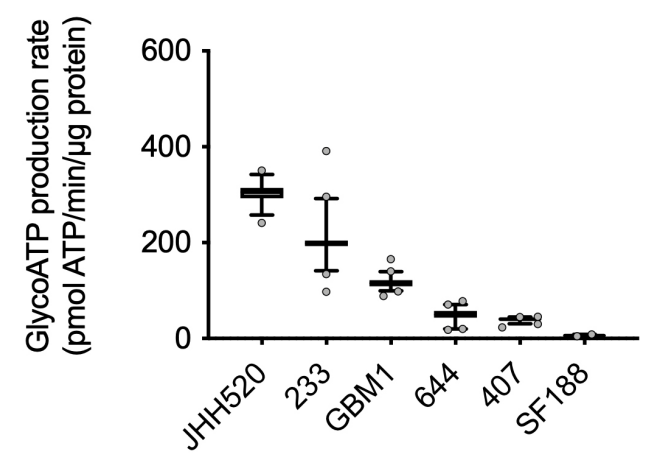

Fig. 1. Glioma stem cells reflect a wide range of total (A), as well as mitochondrial (B) and glycolytic (C) ATP production rates, as assessed by extracellular flux analyses on living cells. However, the range of ratios of glycolytic to mitochondrial ATP productions rates is relatively narrow. The two bioenergetic modes equally contributed to total ATP production, as demonstrated by the similarity of glycolytic to mitochondrial ATP ratios among the different cell lines (D).

activity, as assessed by target gene expression profiling (AXIN2) [30]. The current work suggests a correlation between WNT baseline activity and metabolic state. We previously used AXIN2 as a readout to score the signaling pathway activity in our cells and found that GBM1 is a model with moderate WNT activity, while JHH520 is a model with remarkably high WNT activity [20]. Interestingly, both models showed similarly large drops in ATP synthesis rate upon inhibition of canonical WNT signaling despite manifesting different ATP production rates. Our data suggest that anti-WNT directed interventions may be effective independent of baseline WNT activity. The analyzed cell models belonged to different transcriptional subclasses of the tumor, with GBM1 being a classical subtype and JHH520 a mesenchymal subtype [26]. Thus, our findings suggest that novel therapeutic strategies directed against WNT members - or their downstream signal mediators - may be able to overcome therapy resistance due to molecular heterogeneity of glioblastoma (according to Verhaak et al. [32]). That being said, the models also manifest some homogeneity as they are all wildtype for IDH1 and present methylated MGMT promoter, two consensus markers in clinical neuropathology [33]. From a translational point of view, targeting metabolic pathways, such as glycolysis, to overcome chemotherapy resistance in WNT-signaling-dependent tumors is very relevant, as pharmacological inhibitors of glycolysis are already in an advanced stage of development [28]. Inhibitors of glycolysis may therefore serve as an alternative, possibly less adverse, therapeutic strategy to eradicate WNT-driven malignant behavior of glioma. Moreover, as inhibition of glycolysis affects not only ATP production, but also the availability of $\mathrm{NAD}^{+}$as an important co-factor for different types of cancer cells [34], the anti-glycolytic treatment is warranted as a pan-onco-therapy. Our study extends the list of evidence supporting this concept by describing its relevance as partial WNT inhibition strategy, at least in the context of glioma. Recently, we performed a characterization of the glutaminolytic activity of the cell models used here [35]. The data from Koch et al. [35] together with our own results, indicate that -at least in this cell system-there is no correlation between WNT pathway activity and the amplitude of cellular glutaminolysis. Gluaminolysis inhibition therapy may thus be less effective in targeting WNT-driven glioma stemness compared to anti-glycolytic approaches. A thorough analysis of the effect of glutaminase inhibitors on WNT activation is required to verify this hypothesis. 
A
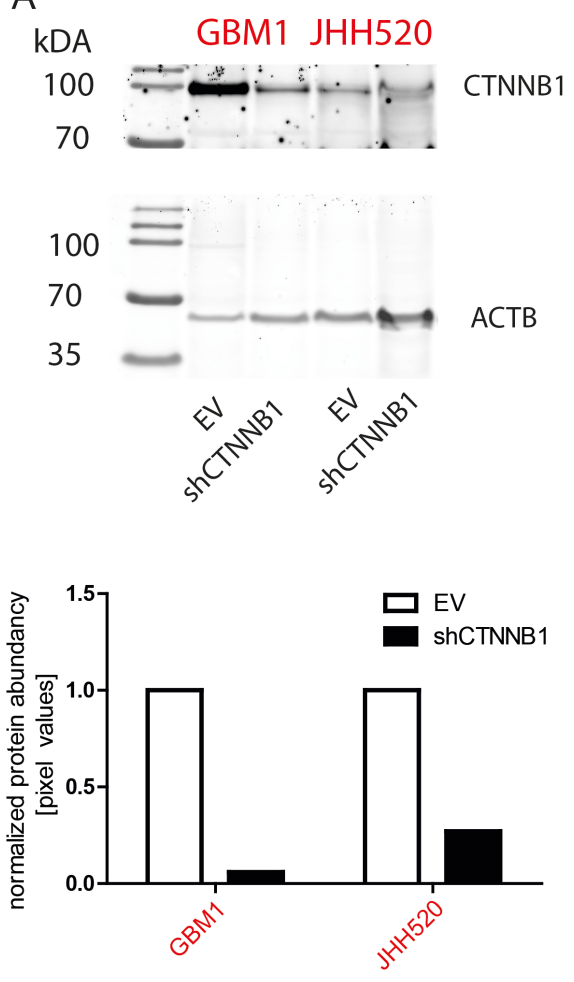

B
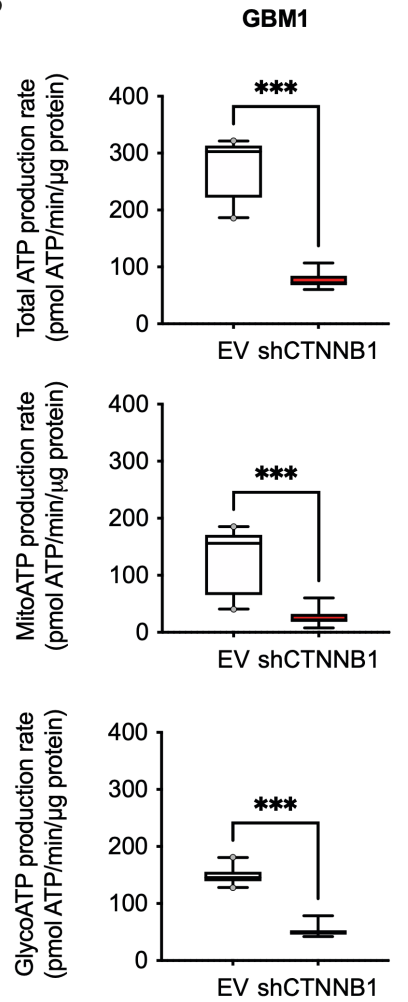
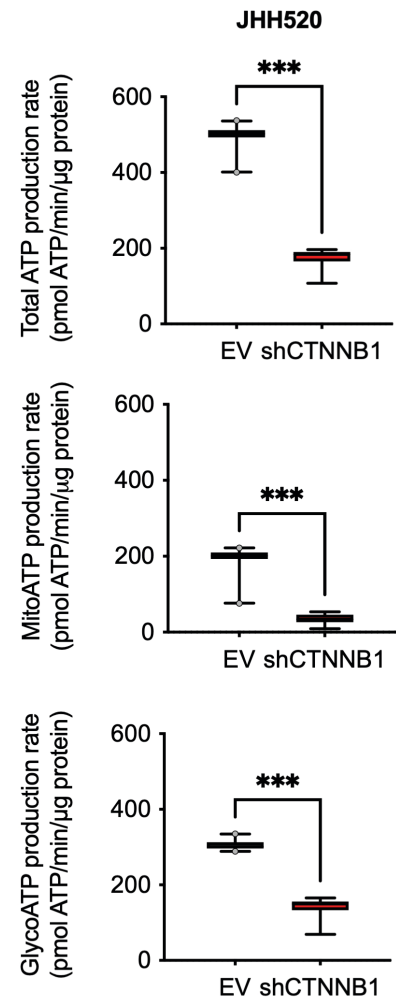

Fig. 2. Establishment of genetic WNT pathway inhibition models via $\beta$-catenin knock-down. (A) Representative Western blot of the WNT model (upper panel) and densitometric protein quantification (lower panel). The visible bands are $\beta$ catenin band ( $-92 \mathrm{kDA})$ and $\beta$ actin ( $-45 \mathrm{kDA}$ ). (B) Effect of CTNNB1 inhibition on total, mitochondrial and glycolytic ATP production. Total ATP production was significantly reduced in both GBM1 and JHH520 GSC lines in which both mitochondrial and glycolytic ATP production rates were significantly reduced as well. ${ }^{* * *}, p<0.001$ for pairwise comparisons between the cell lines as determined by ANOVA and Sidak post-hoc correction. EV, empty vector; shCTNNB1, $\beta$ catenin knockdown.

Surprisingly, our single cell gene expression sequencing data suggest that the WNT-ATP synthesis interaction in GSCs is preferentially mediated through the canonical, CTNNB1-dependent branch of WNT. While we acknowledge that our data is only correlative, thereby less robust as compared to functional data, our observation is in contrast to reports from others in other biological contexts. For instance, WNT5a-mediated activation of non-canonical WNT signaling caused increase in glycolytic activity in melanoma and in cortical neurons [36,37]. Of note, a pioneering work in the field revealed the biological importance of WNT5a to promote GSC maintenance [38,39], but no assessment of the effect of WNT5a on the metabolomics of these cells has been conducted yet.

We are aware of certain limitations of the study that have to be considered carefully when interpreting the data: (1) The gene engineering technology used: the lentiviral approach used in this study cannot exclude that the observed metabolic phenotype is secondary to processes upstream to $\beta$-catenin inhibition. Such processes might be related to the fact that the lentiviral integration happens at an uncontrolled location in the genomic DNA of the target cells, thereby limiting the standardization of our model. Nevertheless, this is unlikely, as the effects were visible in all tested GSC models. In addition, in all tested models, we did not observe a clear trend of altered cell growth upon RNA interference (data not shown). We acknowledge that the use of a second antisense construct directed against CTNNB1 mRNA could support our hypothesis that the metabolic changes are directly related to the inhibition of canonical WNT signaling. (2) Insufficient diversity: Our data present a small number of cell models, especially when hypothesizing that the effect is not restricted to any molecular subtype of glioblastoma. However, since our cell models are well accepted and replicate multiple molecular and cellular features of glioblastoma [26], we believe that the conclusions of the study are substantiated. To benchmark the clinical relevance of our study, in vivo validation in xenograft models would be of great advantage. Optimally, such attempts should be conducted in immunocompetent glioma mouse models, as we recently described [40], to mimic a complete tumor microenvironment. This seems particularly relevant as WNT signaling in cancer cells has been identified as a strong mediator of tumor immune evasion $[41,42]$. 

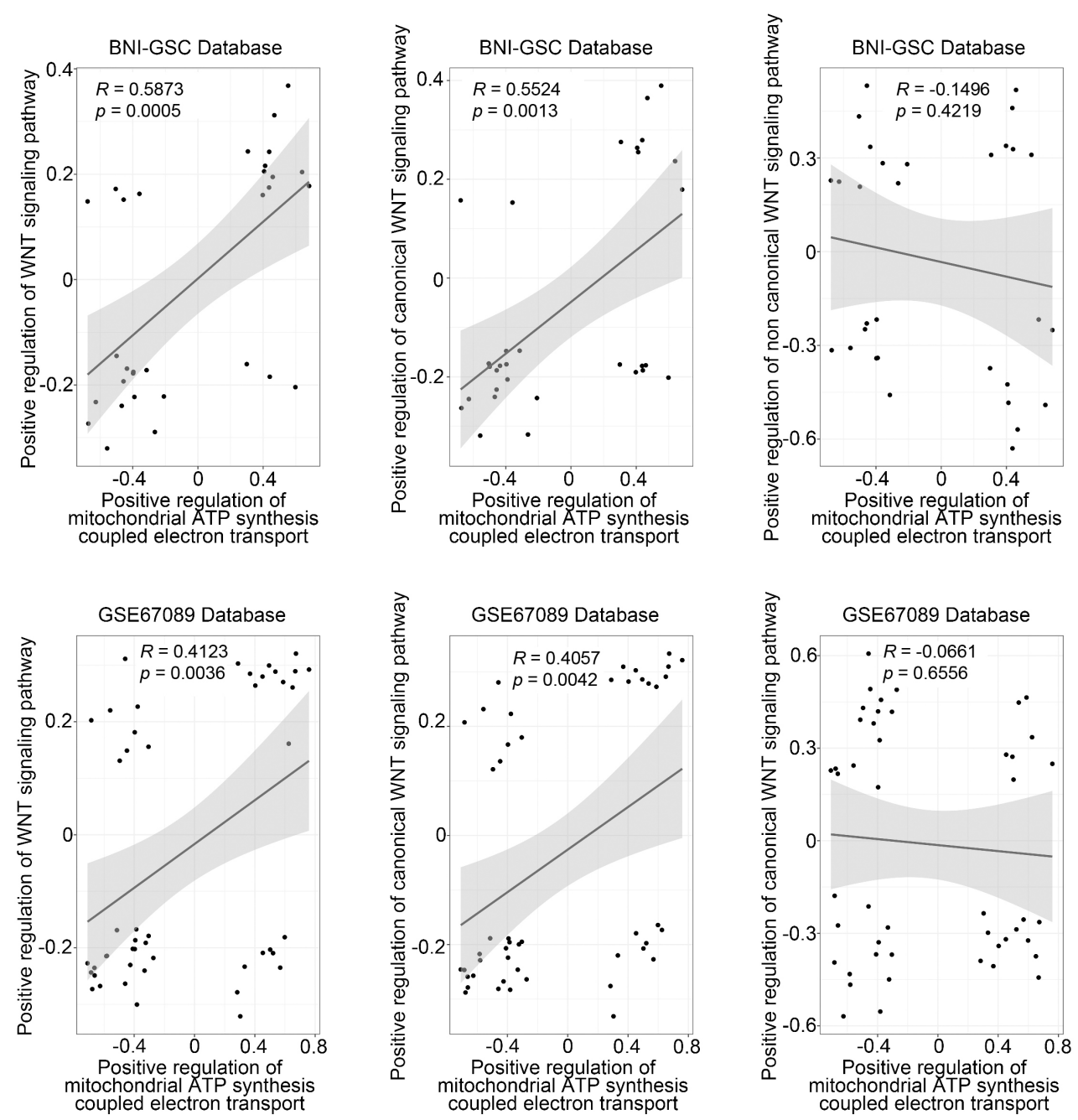

Fig. 3. Correlation analysis of gene expression in GSCs from two independent single cell RNA sequencing datasets (BNI-GSC and GSE67089). There was a strong positive correlation between the expression of ATP synthesis related genes and canonical (CTNNB1dependent) WNT signaling genes. However, the expression of non-canonical WNT genes (CTNNB1 independent pathway) did not correlate with the expression of ATP synthesis-related genes. We also found a positive correlation between the expression of all WNT branches (WNT signaling pathway) and ATP synthesis genes.

\section{Conclusions}

Taken together, our data indicate that metabolic dependency of tumor stem cells are at least in part a result of the aberrant or constitutive activation of phylogenetically conserved canonical WNT signaling. Thus, targeting metabolic pathways is a promising strategy to overcome the resistance to therapy of molecular heterogeneous tumors, such as glioblastoma, as well as an attractive route to overcome the hurdles of clinical translation of hitherto developed anti-tumor stem cell drugs.

\section{Author contributions}

$\mathrm{MO}, \mathrm{MH}$ and UK conceived and designed the experiments; $\mathrm{MO}$ and $\mathrm{MH}$ performed the experiments; $\mathrm{OK}, \mathrm{MO}$, GL, WZ analyzed the data; SM, HJS, EIS, CAD, RSC and $\mathrm{DH}$ contributed reagents and materials; UK was adminis- trating the project, $\mathrm{UK}, \mathrm{MO}, \mathrm{MH}$ and $\mathrm{OK}$ write the original draft. All authors reviewed and edited the draft.

\section{Ethics approval and consent to participate}

The study was conducted in accordance with the Declaration of Helsinki, and the protocol was approved by the Ethics Committee of the Medical Faculty of the HeinrichHeine University (Study ID \#5206). The collection of glioma samples was approved by the Beijing Tiantan Hospital institutional review board (IRB) and written informed consent forms were obtained from every patient.

\section{Acknowledgment}

The authors thank E. Petropoulou, AlfaLab, Greece for her support in the beginning of the study. 


\section{Funding}

This study is a result of the funded by Brigitte \& Dr. Konstanze-Wegener Foundation (Dusseldorf, Germany) granted to UDK (Project \#26).

\section{Conflict of interest}

The authors declare no conflict of interest.

\section{Supplementary material}

Supplementary material associated with this article can be found, in the online version, at https://www.imrpre ss.com/journal/FBL/27/1/10.31083/j.fbl2701035.

\section{References}

[1] Takebe N, Miele L, Harris PJ, Jeong W, Bando H, Kahn M, et al. Targeting Notch, Hedgehog, and Wnt pathways in cancer stem cells: clinical update. Nature Reviews Clinical Oncology. 2015; 12: 445-464.

[2] Clevers, H. Wnt $/ \beta$-catenin signaling in development and disease. Cell. 2006; 127: 469-480.

[3] Jung Y, Park J. Wnt signaling in cancer: therapeutic targeting of Wnt signaling beyond $\beta$-catenin and the destruction complex. Experimental \& Molecular Medicine. 2020; 52: 183-191.

[4] Cairns RA, Harris IS, Mak TW. Regulation of cancer cell metabolism. Nature Reviews Cancer. 2011; 11: 85-95.

[5] Blagodatski A, Poteryaev D, Katanaev VL. Targeting the Wnt pathways for therapies. Molecular and Cellular Therapies. 2014; 2: 28.

[6] Ganapathy-Kanniappan S, Geschwind JH. Tumor glycolysis as a target for cancer therapy: progress and prospects. Molecular Cancer. 2013; 12: 152.

[7] Michelakis ED, Sutendra G, Dromparis P, Webster L, Haromy A, Niven E, et al. Metabolic Modulation of Glioblastoma with Dichloroacetate. Science Translational Medicine. 2010; 2: $31 \mathrm{ra3} 4$.

[8] Yuan S, Lu Y, Yang J, Chen G, Kim S, Feng L, et al. Metabolic activation of mitochondria in glioma stem cells promotes cancer development through a reactive oxygen species-mediated mechanism. Stem Cell Research \& Therapy. 2015; 6: 198.

[9] Flavahan WA, Wu Q, Hitomi M, Rahim N, Kim Y, Sloan AE, et al. Brain tumor initiating cells adapt to restricted nutrition through preferential glucose uptake. Nature Neuroscience. 2013; 16: $1373-1382$.

[10] Vlashi E, Lagadec C, Vergnes L, Matsutani T, Masui K, Poulou $\mathrm{M}$, et al. Metabolic state of glioma stem cells and nontumorigenic cells. Proceedings of the National Academy of Sciences. 2011; 108: 16062-16067.

[11] He TC, Spark AB, Rago C, Hermeking H, Zawel L, da Costa LT, et al. Identification of c-MYC as a target of the APC pathway. Science. 1998; 281: 1509-1512.

[12] Dang CV. MYC, Metabolism, Cell Growth, and Tumorigenesis. Cold Spring Harbor Perspectives in Medicine. 2013; 3: a014217.

[13] Lee SY, Jeon HM, Ju MK, Kim CH, Yoon G, Han SI, et al. Wnt/Snail Signaling Regulates Cytochrome C Oxidase and Glucose Metabolism. Cancer Research. 2012; 72: 3607-3617.

[14] Pate KT, Stringari C, Sprowl-Tanio S, Wang K, TeSlaa T, Hoverter NP, et al. Wnt signaling directs a metabolic program of glycolysis and angiogenesis in colon cancer. The EMBO Journal. 2014; 33: 1454-1473.

[15] Yang W, Xia Y, Ji H, Zheng Y, Liang J, Huang W, et al. Nuclear PKM2 regulates $\beta$-catenin transactivation upon EGFR activation. Nature. 2011; 480: 118-122.
[16] Dong C, Yuan T, Wu Y, Wang Y, Fan TM, Miriyala S, et al. Loss of FBP1 by Snail-Mediated Repression Provides Metabolic Advantages in Basal-like Breast Cancer. Cancer Cell. 2013; 23: 316-331.

[17] Lamb R, Bonuccelli G, Ozsvári B, Peiris-Pagès M, Fiorillo M, Smith DL, et al. Mitochondrial mass, a new metabolic biomarker for stem-like cancer cells: Understanding WNT/FGF-driven anabolic signaling. Oncotarget. 2015; 6: 30453-30471.

[18] Lagathu C, Christodoulides C, Virtue S, Cawthorn WP, Franzin C, Kimber WA, et al. Dact1, a nutritionally regulated preadipocyte gene, controls adipogenesis by coordinating the Wnt $/ \beta$-catenin signaling network. Diabetes. 2009; 58: 609-619.

[19] Willert K, Brown JD, Danenberg E, Duncan AW, Weissman IL, Reya $\mathrm{T}$, et al. Wnt proteins are lipid-modified and can act as stem cell growth factors. Nature. 2003; 423: 448-452.

[20] Kahlert UD, Suwala AK, Koch K, Natsumeda M, Orr BA, Hayashi M, et al. Pharmacologic Wnt Inhibition Reduces Proliferation, Survival, and Clonogenicity of Glioblastoma Cells. Journal of Neuropathology \& Experimental Neurology. 2015; 74: 889-900.

[21] Wang Y, Wang M, Wei W, Han D, Chen X, Hu Q, et al. Disruption of the EZH $2 / \mathrm{miRNA} / \beta$-catenin signaling suppresses aerobic glycolysis in glioma. Oncotarget. 2016; 7: 49450-49458.

[22] Vallée A, Lecarpentier Y, Guillevin R, Vallée JN. Thermodynamics in Gliomas: Interactions between the Canonical WNT/B-Catenin Pathway and PPAR Gamma. Frontiers in Physiology. 2017; 8: 352.

[23] Xu X, Wang L, Zang Q, Li S, Li L, Wang Z, et al. Rewiring of purine metabolism in response to acidosis stress in glioma stem cells. Cell Death \& Disease. 2021; 12: 277.

[24] Suwala AK, Hanaford A, Kahlert UD, Maciaczyk J. Clipping the Wings of Glioblastoma: Modulation of WNT as a Novel Therapeutic Strategy. Journal of Neuropathology \& Experimental Neurology. 2016; 75: 388-396.

[25] Stupp R, Mason WP, van den Bent MJ, Weller M, Fisher B, Taphoorn MJ, et al. Radiotherapy plus concomitant and adjuvant temozolomide for glioblastoma. The New England journal of Medicine. 2005; 352: 987-996.

[26] Nickel AC, Picard D, Qin N, Wolter M, Kaulich K, Hewera M, et al. Longitudinal stability of molecular alterations and drug response profiles in tumor spheroid cell lines enables reproducible analyses. Biomedicine \& Pharmacotherapy. 2021; 144: 112278.

[27] Zhai Y, Li G, Li R, Chang Y, Feng Y, Wang D, et al. SingleCell RNA-Sequencing Shift in the Interaction Pattern Between Glioma Stem Cells and Immune Cells During Tumorigenesis. Frontiers in Immunology. 2020; 11: 581209.

[28] Lund J, Ouwens DM, Wettergreen M, Bakke SS, Thoresen GH, Aas V, et al. Increased Glycolysis and Higher Lactate Production in Hyperglycemic Myotubes. Cells. 2019; 8: 1101.

[29] Selig JI, Ouwens DM, Raschke S, Thoresen GH, Fischer JW, Lichtenberg A, et al. Impact of hyperinsulinemia and hyperglycemia on valvular interstitial cells - A link between aortic heart valve degeneration and type 2 diabetes. Biochimica et Biophysica Acta. Molecular Basis of Disease. 2019; 1865: 2526 2537.

[30] Mehta R, Birerdinc A, Hossain N, Afendy A, Chandhoke V, Younossi $\mathrm{Z}$, et al. Validation of endogenous reference genes for qRT-PCR analysis of human visceral adipose samples. BMC Molecular Biology. 2010; 11: 39.

[31] Garnier D, Renoult O, Alves-Guerra MC, Paris F, Pecqueur C. Glioblastoma Stem-Like Cells, Metabolic Strategy to Kill a Challenging Target. Frontiers in Oncology. 2019; 9: 118.

[32] Verhaak RG, Hoadley KA, Purdom E, Wang V, Qi Y, Wilkerson $\mathrm{MD}$, et al. Integrated genomic analysis identifies clinically relevant subtypes of glioblastoma characterized by abnormalities 
in PDGFRA, IDH1, EGFR, and NF1. Cancer Cell. 2010; 17: $98-110$.

[33] Louis DN, Perry A, Wesseling P, Brat DJ, Cree IA, FigarellaBranger D, et al. The 2021 WHO Classification of Tumors of the Central Nervous System: a summary. Neuro-oncology. 2021; 23: $1231-1251$

[34] Luengo A, Li Z, Gui DY, Sullivan LB, Zagorulya M, Do BT, et al. Increased demand for $\mathrm{NAD}^{+}$relative to ATP drives aerobic glycolysis. Molecular Cell. 2021; 81: 691-707.e6.

[35] Koch K, Hartmann R, Tsiampali J, Uhlmann C, Nickel A, He $\mathrm{X}$, et al. A comparative pharmaco-metabolomic study of glutaminase inhibitors in glioma stem-like cells confirms biological effectiveness but reveals differences in target-specificity. Cell Death Discovery. 2020; 6: 20.

[36] Cisternas P, Salazar P, Silva-Álvarez C, Barros LF, Inestrosa NC. Wnt5a Increases the Glycolytic Rate and the Activity of the Pentose Phosphate Pathway in Cortical Neurons. Neural Plasticity. 2016; 2016: 9839348.

[37] Sherwood V, Chaurasiya SK, Ekstrom EJ, Guilmain W, Liu Q,
Koeck T, et al. WNT5a-mediated $\beta$-catenin-independent signalling is a novel regulator of cancer cell metabolism. Carcinogenesis. 2014; 35: 784-794.

[38] Hu B, Wang Q, Wang YA, Hua S, Sauvé CG, Ong D, et al. Epigenetic Activation of WNT5a Drives Glioblastoma Stem Cell Differentiation and Invasive Growth. Cell. 2016; 167: 12811295.e18.

[39] Binda E, Visioli A, Giani F, Trivieri N, Palumbo O, Restelli S, et $a l$. Wnt5a Drives an Invasive Phenotype in Human Glioblastoma Stem-like Cells. Cancer Research. 2017; 77: 996-1007.

[40] Semenkow S, Li S, Kahlert UD, Raabe EH, Xu J, Arnold A, et al. An immunocompetent mouse model of human glioblastoma. Oncotarget. 2017; 8: 61072-61082.

[41] El-Sahli S, Xie Y, Wang L. Liu S. Wnt Signaling in Cancer Metabolism and Immunity. Cancers. 2019; 11: 904.

[42] Spranger S, Bao R, Gajewski TF. Melanoma-intrinsic $\beta$-catenin signalling prevents anti-tumour immunity. Nature. 2015; 523: 231-235. 